\title{
A CLINICAL STUDY OF PARTIAL OCCLUSION THERAPY IN MANAGEMENT OF AMBLYOPIA IN CHILDREN AGED 5 YEARS TO 15 YEARS
}

Ponnusamy Thiyagarajan ${ }^{1}$, Sudalaiyandi Ganapathirajesh', Mohamed Ali Ayeshatasneem ${ }^{3}$, Noorul Hidaya ${ }^{4}$, Sundaram Anitha 5 , Subbaiah Velusamy Manonmani6

1 Professor, Department of Ophthalmology, Madurai Medical College.

${ }^{2}$ Associate Professor, Department of Ophthalmology, Madurai Medical College.

${ }^{3}$ Senior Resident, Department of Ophthalmology, Madurai Medical College.

${ }^{4}$ Junior Resident, Department of Ophthalmology, Madurai Medical College.

5Junior Resident, Department of Ophthalmology, Madurai Medical College.

6Junior Resident, Department of Ophthalmology, Madurai Medical College.

ABSTRACT
BACKGROUND
Surprisingly $30 \%$ of Indian blinds lose their sight before the age of 20 years, which makes early detection and treatment of visual
impairment for children mandatory. School going children, therefore, form an important large target group and school vision
screening plays an important part in early detection of amblyopia and institution of appropriate therapy, which is of immense value
towards preventing the development of lifelong visual morbidity. The concept of "critical period" which explains the reversibility of
amblyopia at younger age groups when the brain and visual system are immature and connections between neurons are still being
formed and stabilised, stresses the importance of introducing the treatment at an early age thereby enhancing the visual outcome.

\section{MATERIALS AND METHODS}

This clinical study of partial occlusion therapy in the management of amblyopia was conducted among 50 school going children aged 5 years to 15 years in our area to determine the prevalence of amblyopia. Both male and female patients were taken up for study. Children with decreased visual acuity, complaints of asthenia and deviation of the eye were thoroughly examined. Their refractive status, anterior segment and fundus evaluation was done. A diagnosis of amblyopia was made when the child has asymmetric redu ced visual acuity for which there is no identifiable cause made out during a physical examination. They were further grouped into moderate and severe. 'Mild' amblyopia is often classified as being visual acuity of $6 / 9$ to 6/12, 'moderate' amblyopia as being worse than 6/12 to 6/36, and 'severe' amblyopia as being worse than 6/36. A few number of the patients who presented with the temporal pallor of the disc were subjected to CT scan to rule out any organic lesion. After refractive correction with spectacles, depending on the severity of amblyopia, children with moderate amblyopia were advised occlusion for three hours per day and children with severe amblyopia were advised occlusion for six hours per day. They were followed up after three months. However, a very limited number of patients who failed to improve even after six months were referred for alternate treatment options.

\section{RESULTS}

There is a significant difference in the outcomes with age as a factor and patients with normal posterior segment showed better results in three months than patients with the temporal pallor of the disc; however, the tessellated fundus being a confounding factor.

\section{CONCLUSION}

Role of community ophthalmology is a keystone in the prevention of development of amblyopia.

\section{KEYWORDS}

Amblyopia, School Health, Asthenia.

HOW TO CITE THIS ARTICLE: Thiyagarajan P, Ganapathirajesh S, Ayeshatasneem MA. et al. A clinical study of partial occlusion therapy in management of amblyopia in children aged 5 years to 15 years. J. Evolution Med. Dent. Sci. 2016;5(76):5613-5616,

DOI: $10.14260 /$ jemds/2016/1267

\section{INTRODUCTION}

Emmetropia and orthophoria of both eyes are mandatory for the foundation of Binocular Single Vision (BSV). BSV encompasses a point to point normal correspondence of the

Financial or Other, Competing Interest: None.

Submission 17-08-2016, Peer Review 09-09-2016,

Acceptance 16-09-2016, Published 20-09-2016.

Corresponding Author:

Dr. Ponnusamy Thiyagarajan,

Eye Department OP No. 50,

Govt. Rajaji Hospital,

No. 1, Panagal Road, Madurai-625020,

Tamilnadu.

E-mail: rajanophthal@gmail.com

DOI: $10.14260 /$ jemds/2016/1267 two retinae with foveae being the principle corresponding points. In spite of the normal anatomical development of both eyes, interruption of physiological development in visual acuity of one eye can lead to amblyopia. This definition is based solely on visual acuity; however, this definition does not take into account the qualitative differences in vision that an amblyopic often experiences resulting in poorer vision than that measured by Snellen's chart. Amblyopia till date remains a diagnosis of exclusion.

Children constitute $35-40 \%$ of the general population. School going children. ${ }^{1}$, therefore, form an important large target group and school vision screening plays an important part in early detection of amblyopia and institution of appropriate therapy, which is of immense value towards preventing the development of lifelong visual morbidity. 
The time necessary for amblyopia to occur during the critical period. ${ }^{2}$ is shorter for stimulus deprivation amblyopia than for strabismic amblyopia or anisometropic amblyopia. Around the age of two to four weeks, a period of dramatic developmental plasticity. 3 is found to occur when the developing functional and anatomic organisation of the visual system is influenced markedly by visual experiences, which are both uniocular and or binocular. Visually significant conditions like monocular or binocular visual deprivation, anisometropia, strabismus or abnormal visual environment during this period results in significant electrophysiological and anatomic abnormalities in striate cortex and in the lateral geniculate nucleus (LGN).

There occur losses in the number of responsive cells to the deprived eye, a loss of binocularly responsive cells, shrinkage of cells in the LGN laminae serving the deprived eye and significant abnormalities in the response qualities of the cells that remain throughout life. Primate models of amblyopic have repeatedly shown the primary visual cortex (area VI) to be dysfunctional. Functional imaging confirms the presence of processing abnormalities in area VI of the human cortex and also hints at deficits within the higher cortical areas.

In the context of amblyopia, it has often been stated that treatment is ineffective after the age of 8 years. While this may be true for some types of amblyopia, for example, complete unilateral congenital cataract, where treatment must be commenced within a few weeks of birth to be effective, for other causes of amblyopia there is increasing evidence that treatment may be effective beyond this age.

\section{METHODS}

A cross-sectional and time bound study was conducted among 50 children aged 5-15 years attending the OP at Ophthalmology Department, GRH Madurai as well as the School Health Program screening camps. The children who had decreased visual acuity, complaints of asthenia and deviation of eye were thoroughly examined for their refractive status, anterior and posterior segment examination done to determine the prevalence and types of amblyopia among school going children in our area-their improvement after partial occlusion therapy. 4 Patients aged 5-15 years with refractive errors were included in the study. Criteria for exclusion were patients with known cause of reduced visual acuity, patients with myopia greater than spherical equivalent of - 6.00 D, history of previous amblyopia treatment within one year, those who had undergone intraocular surgery, known skin reaction to patch or bandage adhesive and dropouts and noncompliant patients were excluded from final analysis.

\section{Ethical Committee Clearance}

Ethical committee clearance was obtained from Institutional Review Board/Independent Ethics Committee of Govt. Rajaji Hospital.

\section{METHODOLOGY}

A diagnosis of Amblyopia was made and they were further grouped into moderate and severe amblyopia according to their degree of visual loss. A few numbers of the patients who presented with temporal pallor on dilated fundus examination were further subjected to CT scan to rule out a possibility of the organic lesion. A thorough refractive correction. ${ }^{5}$ was done. Patients with high myopia and hypermetropia were excluded from the study. Treatment options like refractive error correction and partial occlusion were discussed with the patients. The duration of occlusion therapy was three hours where the patients were emphasised to do near activity tasks. Compliance to treatment. 6 for more effective results was stressed to both the patient and his/her parents. Depending on the severity of amblyopia, children with moderate amblyopia were advised occlusion for three hours per day and children with severe amblyopia were advised occlusion for six hours per day. The importance of near vision tasks was instructed to the patients. Parents and teachers were asked to motivate the child to wear the occlusion patches.

Patients were explained the importance of treatment of amblyopia, where stress was given on early intervention, selfmotivation and dedicated followup. They were asked to come for followup after three months of occlusion. Significant improvement in visual acuity was seen in younger patients with good compliance which was tested with Snellen's visual acuity chart that showed an improvement in visual acuity by one or two lines. Patients whose visual acuity failed to improve after three months of occlusion therapy were counselled for better compliance and motivation was given to increase the duration of occlusion to six hours a day. However, a very limited number of patients who failed to improve even after six months were referred to an alternate method of treatment and explained about the use of low visual aids, etc.

Patients with a significant amount of ptosis or congenital cataract were explained about the possibility of stimulus deprivation amblyopia and were referred for surgical correction. In each case after analysis of the type of amblyopia, proper counselling was given and the prognosis for each case was discussed with both the parents of the patient and the teachers also.

\section{OBSERVATION AND ANALYSIS}

The number of patients in the younger age group (5-10 years of age) was 32, and those in the older age group (11-15 years of age) were 18 . There were totally 50 patients of whom 26 were boys and 24 were girls. A higher incidence of amblyopia was detected among the younger age group $64 \%$ as compared to the older age group 36\%. A proper understanding of complaints revealed that the patients who came to our OPD mainly complained of visual discomfort (50\%) which was followed by deviation of the eye (30\%) and a few patients had complaints of asthenia. A higher incidence of amblyopia was seen in the rural population (58\%) as compared to the urban population $(42 \%)$. Our study shows moderate amblyopia $(72 \%)$ is more prevalent than severe amblyopia (28\%). The response to occlusion therapy in severe amblyopia is less than moderate amblyopia with some reversibility (Table: 3 ).

A careful analysis of anterior segment in these patients revealed a normal anterior segment in nearly $70 \%$ of the patients and tropias in the remaining $30 \%$. Posterior segment examination revealed normal fundus in the majority of the patients (94\%) and with mild temporal pallor in $4 \%$ of the cases. The patients with temporal pallor were further evaluated with non-invasive imaging studies like CT scan to rule out any brain pathology. CT scan was found to be normal in these patients, helped us in the exclusion of amblyopia of organic aetiology. 
Among the refractive errors prevalent in these patients (Table: 1 ), simple myopia at $20 \%$ and simple hypermetropia at $40 \%$ were commonly seen.

Myopic astigmatism was $8 \%$ more common than hyperopic astigmatism (2\%). The prevalence of myopic among tropias was $10 \%$, exotropia was more commonly seen than esotropia (5\%). The duration of partial occlusion therapy was three to six hours per day.

\begin{tabular}{|c|c|c|c|c|c|c|c|}
\hline Parameter & $\begin{array}{c}\text { Simple } \\
\text { Myopia }\end{array}$ & $\begin{array}{c}\text { Simple } \\
\text { Hypermetropia }\end{array}$ & $\begin{array}{c}\text { Myopic } \\
\text { Astigmatism }\end{array}$ & $\begin{array}{c}\text { Hypermetropic } \\
\text { Astigmatism }\end{array}$ & Esotropia & Exotropia & Total \\
\hline $5-10$ Years & 7 & 12 & 3 & 1 & 3 & 6 & 32 \\
\hline Row $\%$ & $22 \%$ & $38 \%$ & $9 \%$ & $3 \%$ & $9 \%$ & $19 \%$ & $100 \%$ \\
\hline Col\% & $70 \%$ & $60 \%$ & $75 \%$ & $100 \%$ & $60 \%$ & $60 \%$ & $64 \%$ \\
\hline $11-15$ Years & 3 & 8 & 1 & 0 & 2 & 4 & 18 \\
\hline Row $\%$ & $17 \%$ & $44 \%$ & $5 \%$ & $0 \%$ & $11 \%$ & $22 \%$ & $100 \%$ \\
\hline Col\% & $30 \%$ & $40 \%$ & $25 \%$ & $0 \%$ & $40 \%$ & $40 \%$ & $36 \%$ \\
\hline Total & 10 & 20 & 4 & 1 & 5 & 10 & 50 \\
\hline Row $\%$ & $20 \%$ & $40 \%$ & $8 \%$ & $2 \%$ & $10 \%$ & $20 \%$ & $100 \%$ \\
\hline Col\% & $100 \%$ & $100 \%$ & $100 \%$ & $100 \%$ & $100 \%$ & $100 \%$ & $100 \%$ \\
\hline \multicolumn{7}{|r|}{ Table 1: Age wise Stratification of Refractive Status } \\
\hline
\end{tabular}

\begin{tabular}{|c|c|c|c|}
\hline \multicolumn{2}{|c|}{ Post Occlusion Therapy - Third Month } & Not Improved & Total \\
\hline Age & Improved & 8 & 32 \\
\hline $5-10$ Yrs. & 24 & $25.00 \%$ & $100.00 \%$ \\
\hline Row\% & $75.00 \%$ & $53.33 \%$ & $64.00 \%$ \\
\hline Col\% & $68.57 \%$ & 7 & 18 \\
\hline $11-15$ Yrs. & 11 & $38.89 \%$ & $100.00 \%$ \\
\hline Row\% & $61.11 \%$ & $46.67 \%$ & $36.00 \%$ \\
\hline Col\% & $31.43 \%$ & 15 & 50 \\
\hline Total & 35 & $30.00 \%$ & $100.00 \%$ \\
\hline Row\% & $70.00 \%$ & $100.00 \%$ & $100.00 \%$ \\
\hline Col\% & $100.00 \%$ & Table 2: Status after 3 months Post-occlusion Therapy in different Age Groups \\
\hline NH: There is a significant difference in the outcomes with age as a factor AH: There is no significant difference in the outcomes \\
\hline
\end{tabular}

\begin{tabular}{|c|c|c|c|}
\hline \multicolumn{5}{|c|}{ Post-Occlusion Therapy - Third Month } \\
\hline Degree of Amblyopia & Improved & Not Improved & Total \\
\hline Moderate Amblyopia & 30 & 6 & 36 \\
\hline Row\% & $83.33 \%$ & $16.67 \%$ & $700.00 \%$ \\
\hline Col\% & $85.71 \%$ & $40.00 \%$ & 14 \\
\hline Severe Amblyopia & 5 & 9 & $100.00 \%$ \\
\hline Row\% & $35.71 \%$ & $64.29 \%$ & $28.00 \%$ \\
\hline Col\% & $14.29 \%$ & $60.00 \%$ & 50 \\
\hline Total & 35 & 15 & $100.00 \%$ \\
\hline Row\% & $70.00 \%$ & $30.00 \%$ & $100.00 \%$ \\
\hline Col\% & $100.00 \%$ & $100.00 \%$ & \\
\hline
\end{tabular}

\section{DISCUSSION}

Studies have shown that early screening for amblyopia results in better outcomes. Screening for amblyopia begins from infancy. Countries that have instituted early vision screening programs have reduced rates of amblyopia. The American Academy of Paediatrics recommends routine eye screening in newborn period as well in subsequent child visits. High-risk patients, for example, premature babies, children with family history of congenital cataract, malignant tumours like retinoblastoma, those with delayed milestones and genetic illness should be also screened for a comprehensive eye examination. The presence of family history for strabismus and ptosis should alert the paediatrician to refer the patient for a thorough ophthalmic evaluation.
Amblyopia and amblyogenic factors are the commonest target conditions for preschool vision screening and previous reports have stressed that the treatment of amblyopia is not effective after 8 years of age. However, recent reports have supported the view that age is not a barrier to the success of amblyopia therapy. Amblyopia treatment studies (ATS) recently reported improvement in visual acuity in older children also. Hence, the study also includes children of the older age group who were treated with partial occlusion therapy with adequate response in visual potential.

A statistical analysis of the outcome of treatment following three months of occlusion therapy revealed ' $p$ ' value of greater than 0.05 (NH: There is a significant difference in the outcome with age as a factor, $\mathrm{AH}$ : There is no significant difference in the outcome with age as a factor; since p-value is 0.1638 which is 
$>0.05 \mathrm{NH}$ is accepted and $\mathrm{AH}$ is rejected) which concludes the fact that age is a significant factor (Table: 2) in analysing the outcome of treatment of amblyopia.

Even after proper treatment with occlusion therapy, there is a risk of recurrence of amblyopia. ${ }^{7}$ which is around $17 \%$ to 95\%. In a recent study by the PEDIG group, the recurrence rate of amblyopia following therapy was seen in nearly $24 \%$ of the children who were analysed after a year of their treatment. Recurrence rate can be monitored as early as the thirteenth week following discontinuation of therapy. Hence, it is mandatory that proper weaning of therapy is done, like children who were given patching treatment for six hours per day were treated with a weaning therapy of two hours patching per day before discontinuing the treatment to decrease the recurrence rate of amblyopia. The chances of recurrence are always high till the child becomes visually mature i.e. around ten years of age. Hence, a careful monitoring should be done monthly up to the age of 1 year, every 2 months up to the age of 2 years and then every 4 to 6 months up to the age of visual maturity is obtained.

\section{Limitations of the Study}

1. Since the study was time bound, followup of the patients after six months was difficult and hence the analysis of maintenance of the improved visual acuity was not feasible.

2. The incidence and the progression of occlusion amblyopia, an important and a threatening complication of this therapy could not be assessed due to the lack of compliance and time.

\section{CONCLUSION}

The role of critical periods for sensitivity in the development of amblyopia has to be mentioned. 'Critical Period' is the duration of time at which the child's visual system is sensitive to the abnormal input caused by stimulus deprivation, strabismus or significant refractive errors. Another important issue to be assessed is the prognostic significance and variations in different types of amblyopia.

A statistical analysis of the outcome of treatment following three months of occlusion therapy revealed the fact that age is a significant factor in the outcome of treatment for amblyopia. Good compliance to treatment, proper motivation by the parents and most importantly the age of the patient were the reasons for success following treatment.

\section{ACKNOWLEDGEMENTS}

We express our sincere thanks and gratitude to the Dean, Government Rajaji Hospital and Madurai Medical College for permitting us to conduct this study. I express my deep sense of gratitude to HOD of Neurology, and HOD of Paediatrics for evaluation of their patients and their support to this study. I am extremely grateful to all our Assistant Professors and PG Residents for their constant source of cheer and encouragement throughout the study. I thank all my patients who have formed the back bone of my study, without them this work would not have been possible. I am also thankful to paramedical staff of all departments for their concern.

\section{REFERENCES}

1. Desai S, Desai R, Desai NC, et al. School eye health appraisal. Indian Journal of Ophthalmology 1989;37(4):173-5.

2. Daw NW. Critical periods and amblyopia. Arch Ophthalmol 1998;116(4):502-5.

3. Daw NW. Mechanisms of plasticity in the visual cortex. The Friedenwald lecture. Invest Ophthalmol Vis Sci 1994;35(13):4168-79.

4. Singh I, Sachdev N, Brar GS, et al. Part-time occlusion therapy for amblyopia in older children. Indian J Ophthalmol 2008;56(6):459-63.

5. Pediatric Eye Disease Investigator Group. Treatment of anisometropia amblyopia in children with refractive correction. Ophthalmology 2006;113(6):895-903.

6. Al-Zuhair S, Al-Harthi I, Cooymans P, et al. A compliance of amblyopic patients with occlusion therapy: a pilot study. Oman J Ophthalmol 2009;2(2):67-72.

7. Holmes JM, Beck RW, Kraker RT, et al. Risk of amblyopia recurrence after cessation of treatment. J AAPOS 2004;8(5):420-8. 